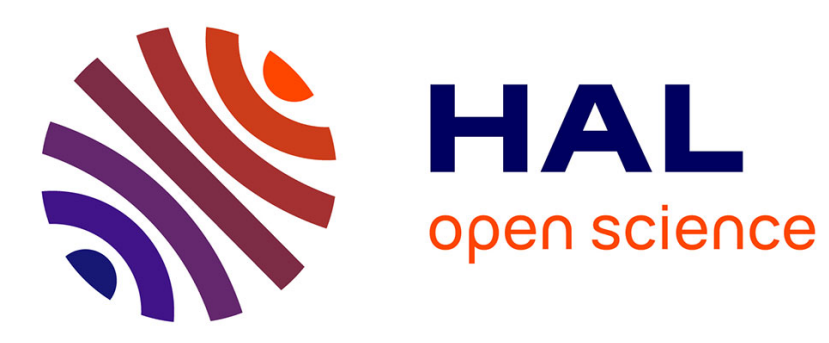

\title{
A 3D Model for Eddy Current Inspection in Aeronautics: Application to Riveted Structures
}

Séverine Paillard, Grégoire Pichenot, Marc Lambert, Hubert Voillaume

\section{To cite this version:}

Séverine Paillard, Grégoire Pichenot, Marc Lambert, Hubert Voillaume. A 3D Model for Eddy Current Inspection in Aeronautics: Application to Riveted Structures. Donald O. Thompson; Dale E. Chimenti. Review of Progress in Quantitative Nondestructive Evaluation, 894, Plenum Press, pp.265 - 272, 2006, 978-0-7354-0399-4. 10.1063/1.2717982 . hal-01103872

\section{HAL Id: hal-01103872 \\ https://hal-centralesupelec.archives-ouvertes.fr/hal-01103872}

Submitted on 15 Jan 2015

HAL is a multi-disciplinary open access archive for the deposit and dissemination of scientific research documents, whether they are published or not. The documents may come from teaching and research institutions in France or abroad, or from public or private research centers.
L'archive ouverte pluridisciplinaire HAL, est destinée au dépôt et à la diffusion de documents scientifiques de niveau recherche, publiés ou non, émanant des établissements d'enseignement et de recherche français ou étrangers, des laboratoires publics ou privés. 


\title{
A 3D MODEL FOR EDDY CURRENT INSPECTION IN AERONAUTIC: APPLICATION TO RIVETED STRUCTURES
}

\author{
S. Paillard ${ }^{1}$, G. Pichenot ${ }^{1}$, M. Lambert ${ }^{2}$ and H. Voillaume ${ }^{3}$ \\ ${ }^{1}$ CEA/LIST, CEA-Saclay, 91191 Gif-sur-Yvette France. \\ ${ }^{2}$ L2S (CNRS-Supélec-UPS), 3 rue Joliot Curie, 91192 Gif-surYvette, France \\ ${ }^{3}$ EADS CCR, DCR/SP/PN, 12 rue Pasteur, 92152 Suresnes France.
}

\begin{abstract}
Eddy current technique is currently an operational tool used for fastener inspection which is an important issue for the maintenance of aircraft structures. The industry calls for faster, more sensitive and reliable NDT techniques for the detection and characterization of potential flaws nearby rivet. In order to reduce the development time and to optimize the design and the performances assessment of an inspection procedure, the CEA and EADS have started a collaborative work aiming at extending the modeling features of the CIVA non destructive simulation plat-form in order to handle the configuration of a layered planar structure with a rivet and an embedded flaw nearby. Therefore, an approach based on the Volume Integral Method using the Green dyadic formalism which greatly increases computation efficiency has been developed. The first step, modeling the rivet without flaw as a hole in a multi-stratified structure, has been reached and validated in several configurations with experimental data.
\end{abstract}

Keywords: Eddy Current Testing, Aeronautic, Fastener Inspection PACS: $41.20 . \mathrm{Jb}-81.70 . \mathrm{Ex}-81.40 . \mathrm{Np}$

\section{INTRODUCTION}

EC technique is currently the operational tool used for fastener inspection which is an important issue for the maintenance of aircraft structures. The industry calls for faster, more sensitive and reliable NDT techniques for the detection and characterization of potential flaws nearby rivets.

In order to reduce the development time, to optimize the design and to evaluate the performances of an inspection procedure, CEA and EADS have started a collaborative work aimed to extend the modeling features of the CIVA non destructive simulation platform to the simulation of multilayer assembly with fasteners.

CIVA is a powerful multi-technique platform for NDT [1]. The developed EC simulation models ([2], [3] and [4]) are mainly based on the volume integral method using the dyadic Green's formalism detailed in [5]. Several examples of CIVA for eddy current testing are presented in Figure 1. A typical configuration of interest is depicted in Fig- 


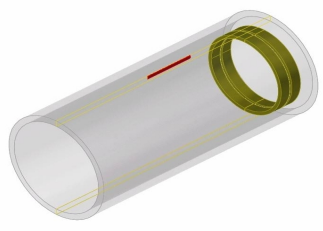

1.a: Bobbin coil placed inside a conducting tube

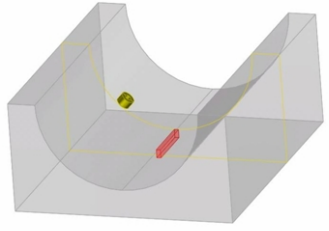

1.b: Bobbin coil placed on a configuration defined by CAD

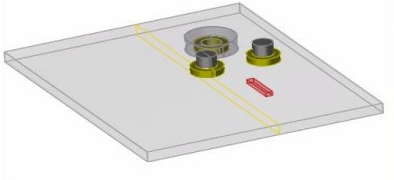

1.c: Three ferrite cores placed

on a conducting slab

FIGURE 1. Representation of several configurations affected by a parallelepiped flaw in the CIVA user interface

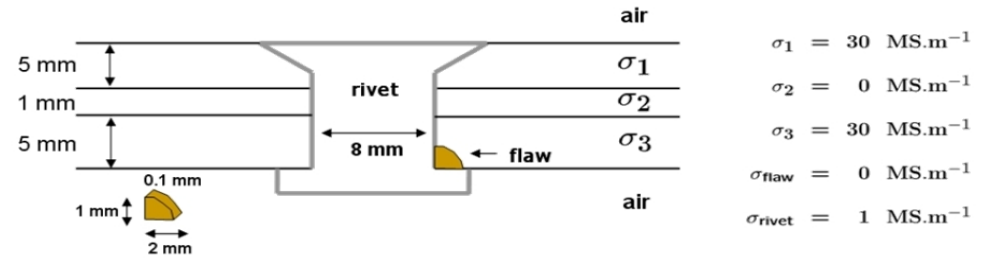

FIGURE 2. Typical aircraft configuration

ure 2. It consists of a layered planar structure with a fastener and a semi-elliptical flaw nearby the lower part of the rivet. The EC probe is moved along the surface, above the fastener assembly.

This paper describes the progress in developing a 3D computer code for fastener modelling based on the volume integral equations which has the capability to quickly predict the response of an eddy current probe to 3D flaws.

\section{THEORETICAL ASPECTS OF THE MULTI-LAYER MODEL}

\section{Modelling steps}

The typical configuration described in Figure 2 can be considered in two steps:

(i) modeling the response of a probe to a layered structure with fastener without flaw as shown in Figure 3.a;

(ii) taking into account the flaw nearby the rivet (see Figure 3.b).

The first step involves the development of multi-layer Green's dyads whereas the second mostly has to cope with a scale issue between a rivet which is a large calculation zone and a small defect nearby. Results of the first step are given below, those of the second one are in progress and will not be detailed here.

\section{Theoretical formulation}

The configuration is described as follows : the space is divided in two air half-spaces 


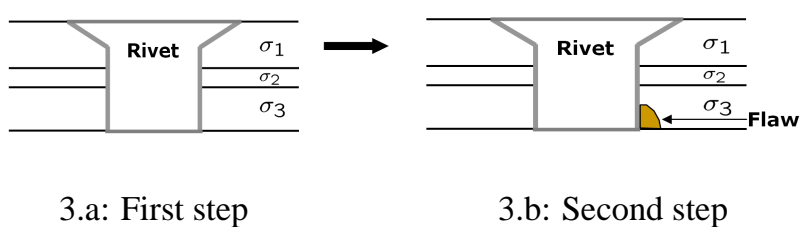

FIGURE 3. Rivet crossing a multi-layer slab with or without a defect nearby

numbered 0 and $N+1$ with, in between, a $N$-layer slab, each layer being numbered $i$ and having a conductivity $\sigma_{i}$ (all materials are supposed to be non magnetic and of air permeability $\left.\mu_{0}\right)$. The slab is affected by a defect of volume $\Omega$ and conductivity $\sigma(\mathbf{r})$ crossing one or more layers (as depicted Figure 2). Let us denote with index $m$ (resp. $n$ ) the first (resp. last) layer affected by the defect $(m<n)$, the later being sliced into as many layers as necessary such as $\Omega=\sum_{k=m}^{n} \Omega_{k}$ (note that, in the case of a rivet crossing the $N$ layers, $m=1$ and $n=N$ ). A time-harmonic source (circular frequency $\omega$ and implied time-dependence $\exp (\mathrm{j} \omega t)$ ) -a coil probe for example-is placed in the upper half-space 0. The so-called vector domain integral formulation of the electric field $\mathbf{E}_{k}(\mathbf{r})$ in the layer $k$ in such a configuration is obtained by application of the Green's theorem onto the diffusive vector wave equation and is given by

$$
\mathbf{E}_{k}(\mathbf{r})=\mathbf{E}_{k}^{(0)}(\mathbf{r})-j \omega \mu_{0} \sum_{l=m}^{n} \int_{\Omega_{l}} \overline{\overline{\mathbf{G}}}_{k l}^{(e e)}\left(\mathbf{r}, \mathbf{r}^{\prime}\right)\left[\sigma_{l}-\sigma\left(\mathbf{r}^{\prime}\right)\right] \mathbf{E}_{l}\left(\mathbf{r}^{\prime}\right) d \mathbf{r}^{\prime} \quad \forall \mathbf{r}^{\prime} \in \Omega_{k}
$$

where $\mathbf{E}_{k}^{(0)}(\mathbf{r})$ is the primary field in the layer $k$ and $\overline{\mathbf{G}}_{k l}^{(e e)}\left(\mathbf{r}, \mathbf{r}^{\prime}\right)$ the electric-electric dyadic Green's functions defined as the field response for a unit point source and solution of

$$
\nabla \times \nabla \times \overline{\overline{\mathbf{G}}}_{k l}^{(e e)}\left(\mathbf{r}, \mathbf{r}^{\prime}\right)-k_{k}^{2} \overline{\overline{\mathbf{G}}}_{k l}^{(e e)}\left(\mathbf{r}, \mathbf{r}^{\prime}\right)=\delta_{k l} \overline{\overline{\mathbf{I}}} \delta\left(\mathbf{r}-\mathbf{r}^{\prime}\right)
$$

In the above equations $k, l$ denote the index of the layer of the observation $\mathbf{r}$ and of the source ' $\mathbf{r}$ ' point, respectively, $\overline{\overline{\mathbf{I}}}$ is the unit dyadic and $\delta_{k l}$ stands for the Kronecker delta. $k_{l}$ is the wave number in the $l^{\text {th }}$ layer defined as $k_{l}^{2}=\mathrm{j} \omega \mu_{0} \sigma_{l}$. The Green's dyad satisfies the appropriate boundary conditions at the interfaces between the different layers in the same way as the electric fields do. The response of the probe is given by its impedance variation is obtained via the reciprocity theorem, where $I_{0}$ is the feeding current of the probe, as

$$
I_{0}^{2} \Delta Z=\sum_{l=m}^{n} \int_{\Omega_{l}}\left[\sigma_{l}-\sigma(\mathbf{r})\right] \mathbf{E}_{l}^{(0)}(\mathbf{r}) \cdot \mathbf{E}_{l}(\mathbf{r}) d \mathbf{r} .
$$

\section{Multi-layer Green's functions}

These Green's functions are decomposed in self-coupling terms named $\overline{\overline{\mathbf{G}}}_{i i}$ with $i \in\{1, N\}$, and mutual coupling terms named $\overline{\overline{\mathbf{G}}}_{i j}$ with $(i, j) \in\{1, N\}$ and $i \neq j$. A rivet being through-wall, it is divided in as many parts than the number of layers (here $N)$. In consequence, each part of the rivet is contained in one layer.

An example is given for a three-layered slab $(N=3, n=1$ and $m=3)$ in Figure 4. The rivet illustrated in Figure 4.a is sliced into three parts, each one entirely contained 
in a single layer of conductivity $\sigma_{k}$. The self-coupling terms $\overline{\overline{\mathbf{G}}}_{i, i}$ with $i \in\{1,3\}$ are represented in Figure 4.b and the mutual-coupling terms $\overline{\overline{\mathbf{G}}}_{i, j}$ with $(i, j) \in\{1,3\}$ and $i \neq j$ are represented in Figure 4.c.

For building this multi-layer model, two main improvements have been made:

(i) the planar stratification of the work piece is taken into account by introducing generalized reflection and transmission coefficients at each interface in the Green's dyads;

(ii) the mutual Green's functions are written in explicit analytical expressions [5].

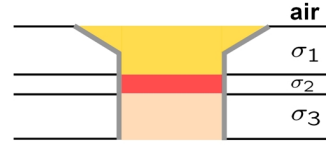

4.a: Rivet sliced in three inhomogeneity zones

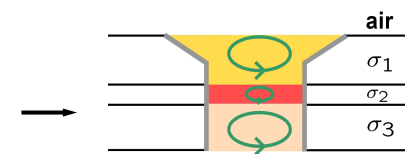

4.b: Self-coupling

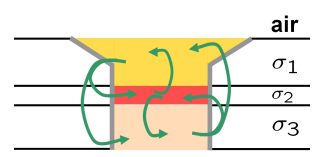

4.c: Mutual coupling

FIGURE 4. Example of a rivet in a three-layered slab

\section{Numerical considerations}

Once the model has been chosen and the equations established, the numerical formulation can be implemented. Equation (1) is discretized using a Galerkin's version of the method of moments where the obstacle $\Omega$ is seen as $N_{\text {cell }}$ parallelepipeded voxels of homogeneous conductivity in which the electric field is taken as constant-valued. This approach leads to a linear system (4)

$$
\left[\begin{array}{c}
\mathbf{E}_{m}^{(0)} \\
\vdots \\
\mathbf{E}_{n}^{(0)}
\end{array}\right]=\left(\mathbf{I}-\left[\begin{array}{ccc}
\Omega_{m, m} & \cdots & \Omega_{m, n} \\
\vdots & \ddots & \vdots \\
\Omega_{n, m} & \cdots & \Omega_{n, n}
\end{array}\right]\right)\left[\begin{array}{c}
\mathbf{E}_{m} \\
\vdots \\
\mathbf{E}_{n}
\end{array}\right]
$$

where $\Omega_{i, i}$ are the electromagnetic self-coupling terms of the $i^{t h}$ region of the sliced rivet onto itself and where $\Omega_{i, j}$ are the mutual coupling terms of the $j^{\text {th }}$ over the $i^{t h}$. In the applications we are interested in, the typical size of the domain $\Omega$ may be more than ten skin-depths which leads to a large number of voxels and to a too large linear system to invert (the memory size can be estimated as $\mathscr{O}\left(9 N_{\text {cell }}^{2}\right)$ ). Taking into account the convolution structure of the integral equation (1) with respect to the two lateral directions via appropriate fast Fourier transforms, an iterative solution of the system allows us to treat larger defects by reducing the memory size to $\mathscr{O}\left(9 N_{\text {cell }}^{4 / 3}\right)$. 


\section{MULTI-LAYER VALIDATIONS}

\section{Experiments}

The comparison between simulation and experimental data is used to validate our approach in all the aspects of the multi-layer model. The simplest planar configuration available is a two layers configuration made of materials with different conductivities - inconel $\left(\sigma_{\text {inconel }}=1 \mathrm{MS} / \mathrm{m}\right)$ and aluminium $\left(\sigma_{\text {aluminium }}=30 \mathrm{MS} / \mathrm{m}\right)$. As depicted in Figure 5, from such a configuration three experiments can be carried out in order to focus and to test each Green's dyads:

(i) A breaking surface hole in the inconel top layer (Figure 5.b). It enable us to set apart the self-coupling term $\overline{\mathbf{G}}_{11}$ in a stratified work piece.

(ii) A hole in the aluminium bottom layer (Figure 5.c). It enable us to set apart the self-coupling term $\overline{\mathbf{G}}_{22}$ in a stratified work piece.

(iii) A through-wall hole in the two-layer work piece (Figure 5.d). The mutual coupling terms, $\overline{\mathbf{G}}_{12}$ and $\overline{\mathbf{G}}_{21}$ can not be isolated from the self-coupling ones, $\overline{\mathbf{G}}_{11}$ and $\overline{\mathbf{G}}_{22}$ and all the Green's dyads are used in this case.

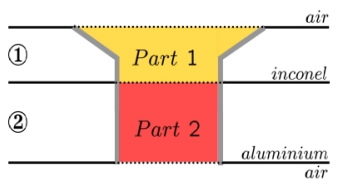

5.a: Sliced rivet in two parts

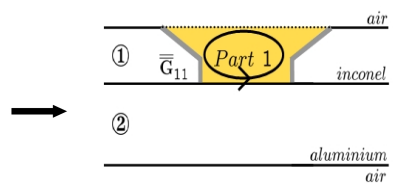

5.b: Part one only

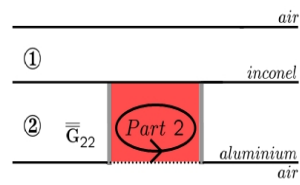

5.c: Part two only

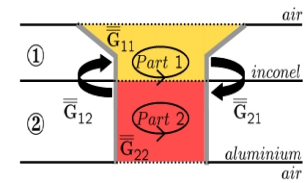

5.d: The two parts

FIGURE 5. Testing the multi-layer Green's dyads with three experiments

To focus on these different aspects, and to avoid errors in rivet shape simulation, the rivet with its typical flat head shape is assumed in all validations to be a cylindrical through-wall hole of air. The three configurations are theoretically depicted in Figure 5 and are practically represented as experiments in the left column of Figure 7.

Several validations tests have been carried out to improve the two approaches handle a fastener in a laminated slab and a multi-layer configuration- of this multi-layer model. For these studies the same air-cored probe is used (an inner radius of $1 \mathrm{~mm}$, an outer radius of $1.6 \mathrm{~mm}$, a lift-off of $0.32 \mathrm{~mm}$ and a height of $2 \mathrm{~mm}$ with 320 turns) and is displaced along the diameter of the hole.

\section{Calibration}

In most industrial applications, the measured EC signal is calibrated over a reference flaw. Preliminary to these validations, a calibration experiment has been made; the reference flaw is a surface breaking notch in an inconel slab with a conductivity of $1 \mathrm{MS} / \mathrm{m}$. The EDM notch is $0.1 \mathrm{~mm}$ in width, $20 \mathrm{~mm}$ in length and $0.93 \mathrm{~mm}$ in depth and the thickness of the slab is $1.55 \mathrm{~mm}$ as shown in Figure 6 (left). The impedance variation 

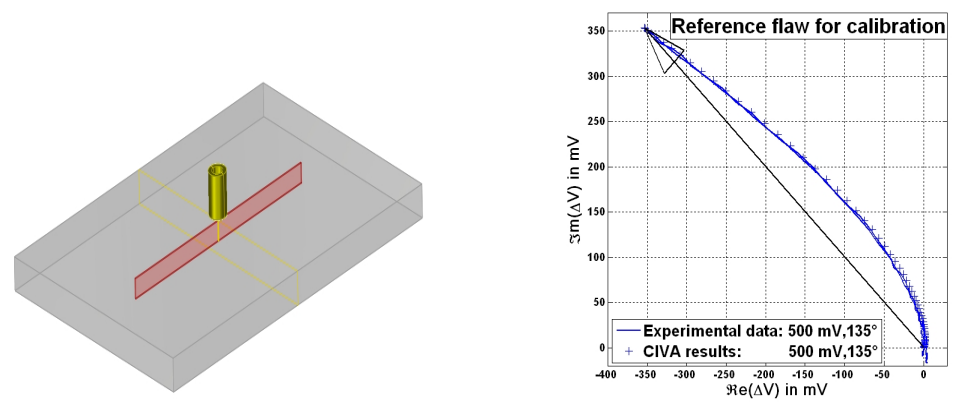

FIGURE 6. Response of the probe to a breaking notch in a slab ( - experimental data, +++ CIVA results)

measured in the impedance plane calibrated at $500 \mathrm{mV}$ and $135^{\circ}$ is presented in Figure 6 (right). 

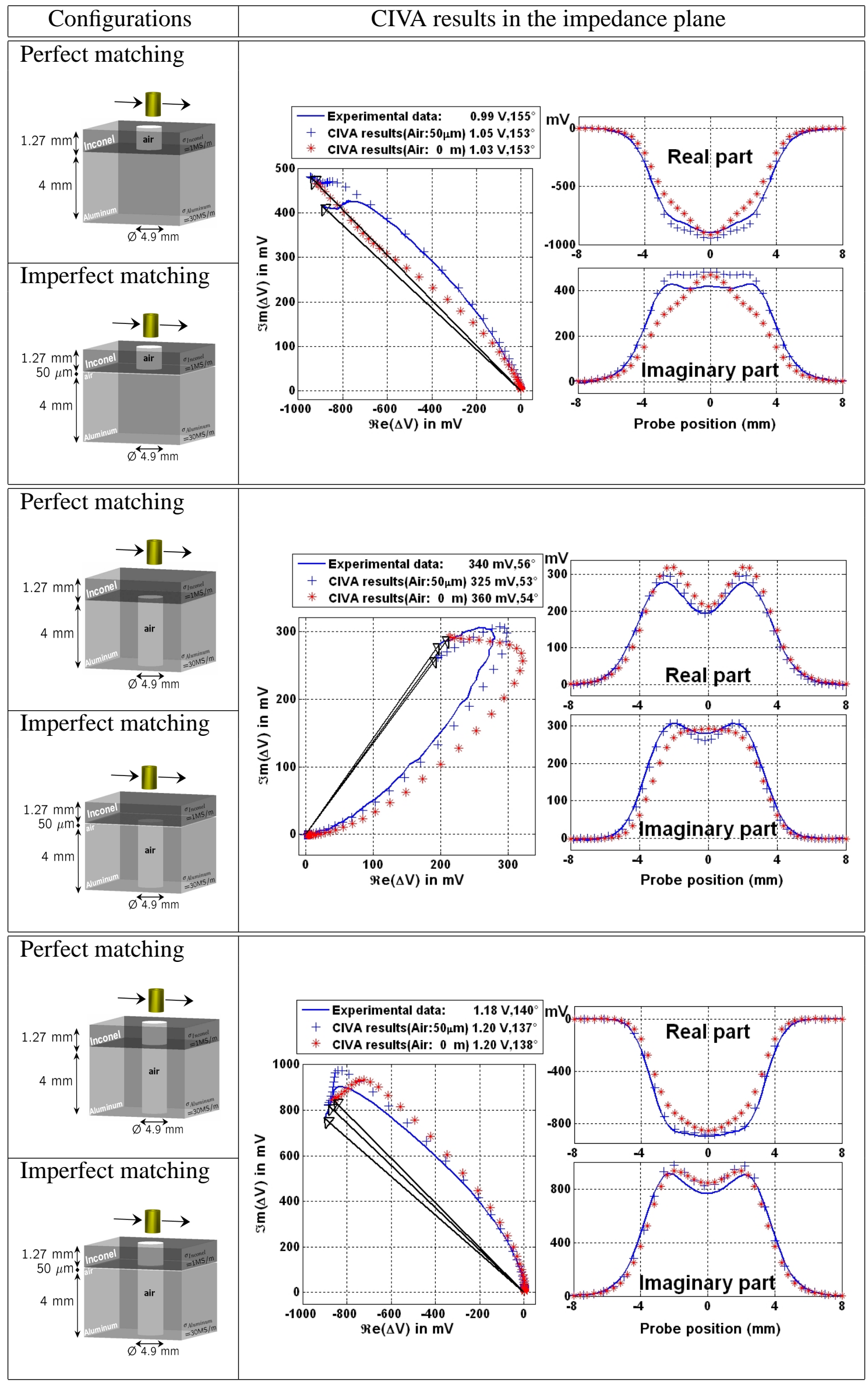

FIGURE 7. Summary of the three configurations with perfect and imperfect matching (- experimental data, ${ }^{* * *}$ CIVA results with perfect matching, +++ CIVA results with imperfect matching) 


\section{Matching slabs influence}

Each experiment theoretically depicted in Figure 5 can be simulated with perfect or imperfect matching between the two slabs.

A perfect matching simulation corresponds exactly to the definition of the experimental configuration. On the contrary, the imperfect matching simulation should correspond to real experimental configuration where the two slabs could not be fastened in perfect fashion leading to the occurrence of a thin air layer in between. A study has been carried out to evaluate the thickness of the layer of air to be taken into account and the best results have been obtained with a thickness of $50 \mu \mathrm{m}$. The results of the multi-layer model with the perfect and the imperfect matching configurations (see in the left column of Figure 7) are represented with respectively stars $\left(^{*}\right)$ and plus (+) in the right column of Figure 7.

\section{Final results of validations}

The results of the perfect matching are not completely satisfactory; even if the agreement for the measurement of the EC signal in the impedance plane between the model and the experimental data is better than $4 \%$ for the amplitude and $2^{\circ}$ in phase, the shapes of the signal are different.

The results of the perfect matching are closer to the experiment. For the hole in the inconel slab, the agreement between the model and the experimental data is better than $6 \%$ for the amplitude and $2^{\circ}$ in phase (first configuration of Figure 7). For the hole in the aluminium slab, the agreement is better than $4 \%$ for the amplitude and $3^{\circ}$ in phase (second configuration of Figure 7). Concerning the through-wall hole, which allows to validate the aspects of the multi-layer model (self and mutual coupling of layers), the agreement is better than $2 \%$ for the amplitude and $3^{\circ}$ in phase (third configuration of Figure 7).

One of the reasons can be that the simulated configuration with perfect matching does not correspond exactly to the reality of the experimental configuration contrary to the imperfect matching configuration.

A comparison with Finite Element is in progress to see the matching slab influence with another method.

\section{CONCLUSION AND PERSPECTIVES}

The extension of the CIVA platform to the simulation of riveted structures is currently in progress. The multi-layer model is now validated, with a good agreement between the model and the experimental data, for a cylindrical through-wall hole in a set of two slabs, a cylindrical hole either in the top slab or in the bottom slab of the stack.

A first milestone has been reached with the development of a model taking into account the presence of a rivet in a layered slab assembly. Validations with experimental data of the 3D model developed here for fastener modeling have been carried out successfully. Work is in progress to calculate the probe response due to the presence in a fastened structure of both a rivet and an embedded flaw located nearby. 


\section{ACKNOWLEDGEMENTS}

This research is supported by the Paris Ile-de-France Region.

\section{REFERENCES}

1. Le Ber L., Calmon P., Sollier T., Mahaut S. and Benoist P., Advances of simulation and expertise capabilities in CIVA platform, in Review of Progress in QNDE Vol. 25, 2006, pp. 684-691.

2. Buvat F., Pichenot G., Prémel D., Lesselier D., Lambert M. and Voillaume H., Eddy current modelling of ferrite-cored probes, in Review of Progress in QNDE Vol. 24, 2005, pp. 463-470.

3. Sollier T., Buvat F., Pichenot G. and Premel D., Eddy current modelling of FerriteCored Probes, application to the simulation of Eddy current signals from surface breaking flaws in austenitic steel, Proc. 16th World Conf. on NDT, Montreal, 2004.

4. Pichenot G., Buvat, F., Maillot V. and Voillaume H., Eddy current modelling for non destructive testing, Proc. 16th World Conf. on NDT, Montreal, 2004.

5. Chew W.C., Waves and Fields in Inhomogeneous Media, Van Nostrand Reinhold, New York, 1990. 